\title{
Poetic of Pasaje Corazón de Jesús building's memory, in the times of the telegraph: Prototype of Video Mapping
}

of the Iberoamerican Society

of Digital Graphics

Medellin | Colombia

\author{
Sandra Calvachi Arciniegas \\ Universidad de Nariño | Colombia | calvachisan@yahoo.es \\ Juliana Bravo Montero \\ Universidad de Nariño | Colombia | julianabravom@hotmail.com \\ Juliana Rosero Jurado \\ Universidad de Nariño | Colombia | julianarjurado@hotmail.com
}

\begin{abstract}
The project is about the elaboration of a Video Mapping projection over a facade's model of the emblematic Pasaje Corazón de Jesús building, located in the city of Pasto, scale 1:15; since a conceptual point of view, through the narrative exploration created from the history of the own building itself in the period of time in which it worked as a telegraph and post office. This play proposes a new way to see and create the memory since the experiences with the purpose of communicates, keep and give value to the place's historical memory and to its poetic.
\end{abstract}

Keywords: Video Mapping; Poetic; Memory; Telegraph; Pasaje Corazón de Jesús building.

\section{INTRODUCCIÓN}

En este estudio se presenta parte de la investigación "La poética del lugar a través de la creación audiovisual: Fase experimental de Video Mapping sobre el pasaje Pueyo de Val en la Plaza de Nariño en Pasto", financiado por la Vicerrectoría de Investigaciones e Interacción Social de la Universidad de Nariño.

El proyecto se enmarca en el rescate de la memoria de edificio Pasaje Corazón de Jesús a través de un Video Mapping, cuyo proceso metodológico de creación propone una forma de ver y crear la memoria a partir de la poética, reconstruida desde las experiencias y vivencias adquiridas por quienes relataron testimonios de la época.

La obra se enfoca en la indagación de hechos históricos acontecidos en el periodo de tiempo en el cual el inmueble alojó las oficinas de telégrafos, es decir entre los años 1930 y 1963, obtenidos a través de documentación y por medio de entrevistas a testigos. Estos hechos cuentan con muy poca visibilidad, ya que no se encuentran mayores registros en archivos, por ello se busca evidenciar dicha memoria a través del proceso de creación de la obra audiovisual.

De esta forma, se abren los límites, posibilitando reconocer dicha memoria y ponerla en evidencia a través de la narrativa, plasmada en una obra audiovisual como el Video Mapping, un espectáculo atractivo para la gente; transmitiendo dicho conocimiento hacia un público más extenso y generando un impacto en quien vive la experiencia, es decir propiciando el fenómeno de repercusión y evidenciando estos hechos sobre la fachada, con el fin de resaltar su poética (Bachelard, G. 1958).

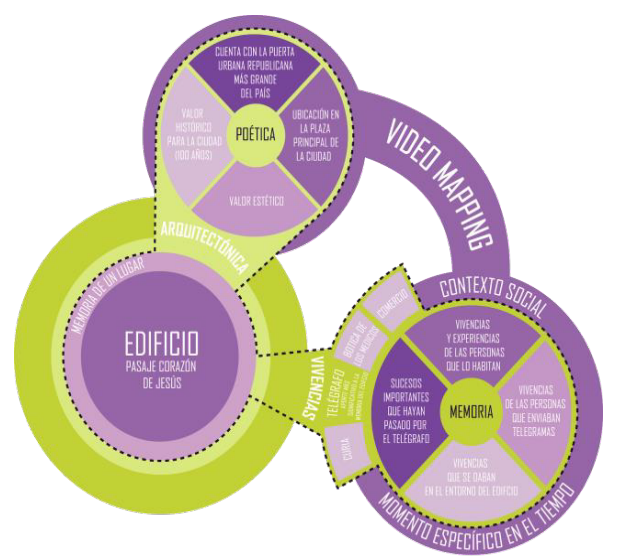

LA MEMORIA A PARTIR DE LAS VIVENCIAS

Figura 1: Explicación del concepto general del proyecto.

En arquitectura, usualmente se trazan límites que condicionan el concebir y percibir la memoria encasillándola en el concepto de patrimonio y en su papel urbano; esta investigación propone una forma ver y crear la memoria de un lugar a partir de experiencias sensoriales y vivencias adquiridas a través de quienes lo habitan, generando así, una relación entre el cuerpo y el espacio, "Las experiencias sensoriales pasan a integrarse a través 
del cuerpo, o, mejor dicho, en la misma constitución del cuerpo y el modo de ser humano." (como se cita en Camilo, 2016). A través de dichas experiencias se va acumulando la noción de memoria.

Se considera que la monumentalidad del edificio Pasaje Corazón de Jesús sea percibida a partir de las experiencias acumuladas en el periodo de tiempo en que funcionó como oficinas de telégrafo y su contexto. Dichas vivencias condicionadas por el momento y contexto van formando parte de su memoria, "(...) la memoria es la representación del pasado concentrada en un objeto". (Sztulwarkk, 2005. p.2). Este inmueble es un ejemplo de lo mencionado, al ser testigo de las vivencias de varias personas del mundo a través de telegramas, lo cual supone que la memoria del edificio teniendo exactamente el uso del telégrafo, fue lo que condicionó las vivencias de las personas y de igual forma estas influyeron en la percepción del edificio en la memoria colectiva. "La arquitectura es una forma expresiva que dice cosas que van más allá de la función; asume aspectos metafóricos y simbólicos que persisten por encima de su uso inmediato, y llegan a ser parte de una historia que nos pertenece." (Botta, 2014. P. 97), entendiendo así que la suma de todos estos aspectos es lo que permite conectarse con el pasado.

\section{EL RESCATE DE LA MEMORIA DE UNA CIUDAD OLVIDADA A TRAVÉS DEL VIDEO MAPPING}

Para entender el contexto en el periodo de tiempo a estudiar comprendido entre los años 1925 y 1971 , hay que tener en cuenta que, a diferencia de otras ciudades, Pasto tuvo una condición con cierta desventaja en cuanto a reconocimiento y apoyo para su desarrollo general. Es por esta razón que la permanencia de las oficinas del telégrafo en el edificio entre los años 1930 y 1963, tuvo un gran impacto en las condiciones sociales de la comunidad y su desarrollo en cuanto a comunicación. Por otro lado, estas instalaciones se localizan en una de las principales y más importantes edificaciones de la ciudad, con una estética impecable, además de ser una representativa puerta urbana republicana que se conserva en el país, la cual se abre hacia la plaza principal de la ciudad. Por todo lo anteriormente mencionado se privilegia el enfoque en este inmueble y en esta época en especial, con el fin de aprovechar estos dos aspectos importantes, que al unirse logran mayor potencial.

El edificio Pasaje Corazón de Jesús fue construido desde el año 1925 a 1928, por el Arquitecto Lucindo Espinosa, con el apoyo de la gestión del obispo Pueyo de Val. El inmueble pertenece al estilo republicano, con ornamentos de estilo neoclásico, enmarcando la plaza principal junto con otros inmuebles de gran importancia patrimonial, como la Iglesia de San Juan, siendo la más antigua de la ciudad, o como la casa de Don Lorenzo. Todo esto lo lleva a considerarse un inmueble patrimonial a nivel nacional. "El pasaje corazón de Jesús se constituye como un hito arquitectónico y urbano en el centro de la ciudad, siendo dentro de la plaza, junto al templo de San Juan y a la casa de don Lorenzo, el costado más emblemático e importante de este espacio público." (Enríquez, Ordóñez, Morales,

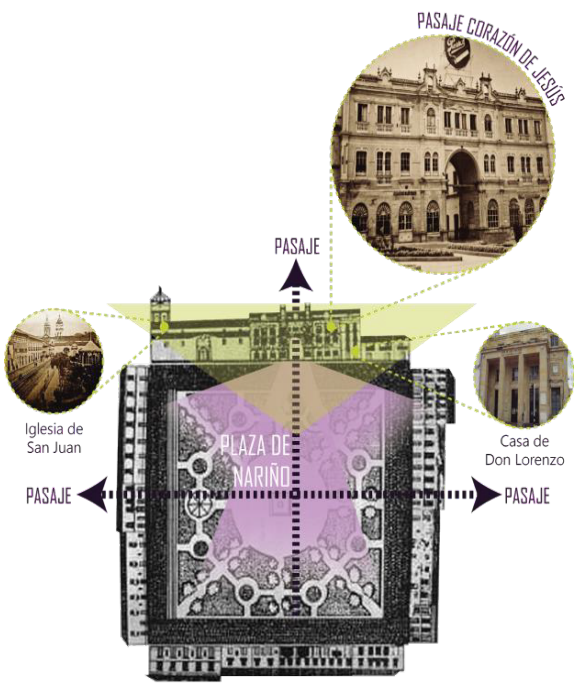

Bolaños, \& Ortega, 2009, p. 49-50). (figura 2).

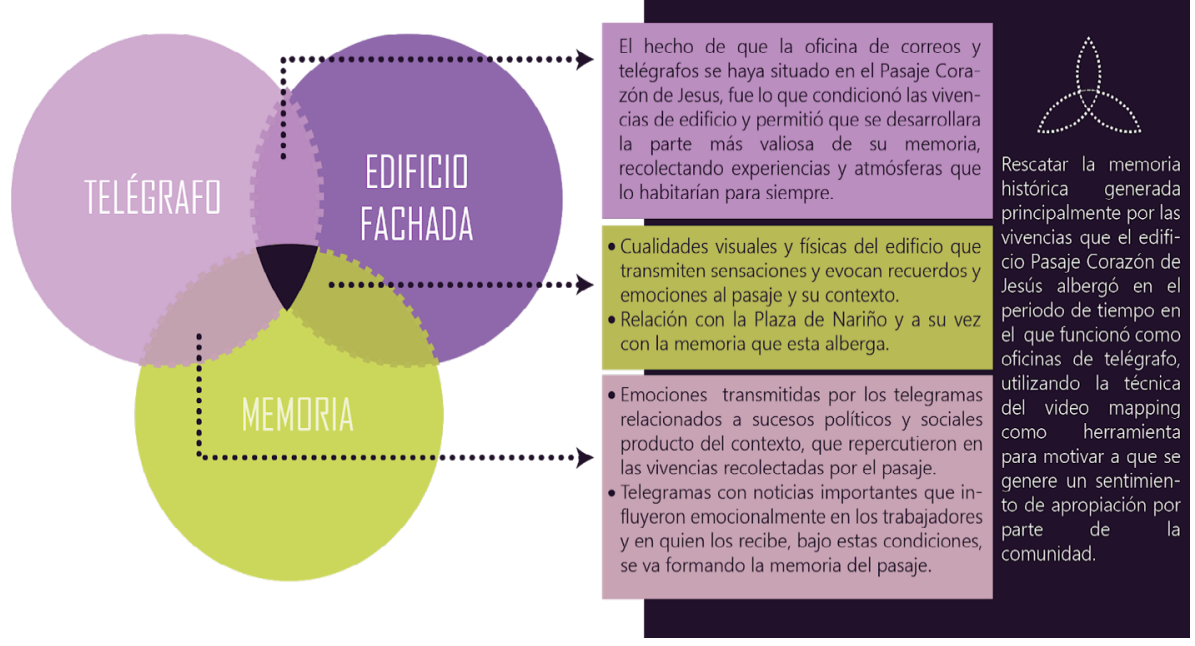

Figura 3: Desglose de las temáticas. 


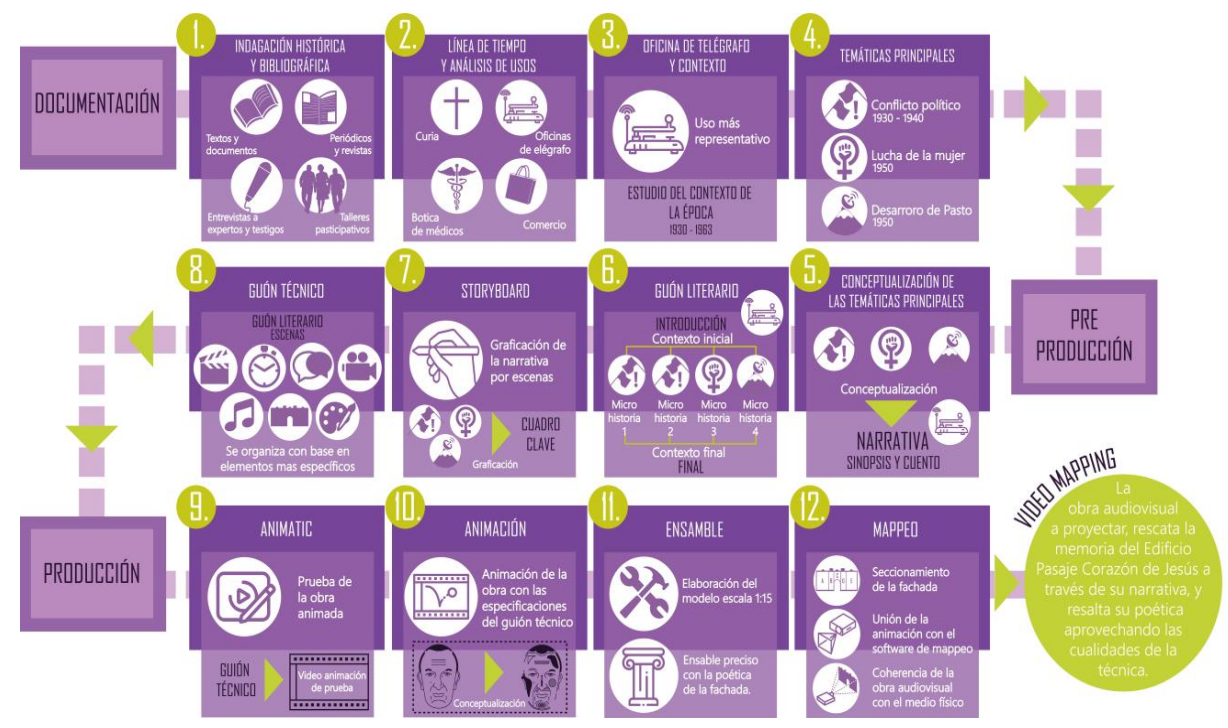

Figura 4: Secuencia metodológica del proyecto.

Para darle visibilidad y rescatar esta memoria, se parte desde la misma ausencia y situación de olvido, aprovechando el potencial que existe en la arquitectura para dar a conocer dicha memoria conceptualmente. Una potente forma de comunicar dicha narrativa es el uso de formatos a gran escala, estrategia que busca propiciar el efectivo rescate de la memoria, como herramienta que fomente la apropiación de la historia y la cultura, cuyo público debe abordar la mayor cantidad de ciudadanos posible, es por ello que la técnica a usar es el Video Mapping; un método llamativo y colosal que no sólo rescata la memoria sino que también evoca recursos patrimoniales físicos resaltando su poética y dejando en evidencia la belleza de cada uno de los elementos que componen la fachada. "La memoria encuentra en la obra de arte un modo silencioso de ser, una condición antropológica que está en condiciones de sugerir auténticas invenciones proyectuales alejadas de imposibles huidas nostálgicas (...)". (Botta, 2014, p.97). De esta forma se busca que el edificio sea visto como un ente vivo que guarda una memoria invaluable (figura 3).

\section{METODOLOGÍA Y RESULTADOS}

El abordaje de la investigación es de tinte cualitativo con énfasis en aspectos fenomenológicos. El proceso se desarrolló en tres grandes etapas, las cuales fueron: la documentación, que abarca la recopilación de información; la preproducción correspondiente a la creación conceptual y la producción, fase en la que se realizó el animatic, la animación, el ensamble de la maqueta y el mapeo de la obra audiovisual a proyectar (figura 4), (tabla 1).

\section{DOCUMENTACIÓN}

\section{INDAGACIÓN HISTÓRICA}

En la búsqueda de la información se identificaron los acontecimientos más destacables de la época de estudio, y para ello se tuvieron en cuenta dos aspectos fundamentales: el primero consistió en la documentación recopilada a través de una ardua indagación en diversas fuentes que se refieren a la historia de Pasto desde diferentes aspectos: textos, libros vídeos, fotografías digitales y físicas, grabaciones, etc. Esta recopilación se realizó a través de diferentes estrategias como: visita a los museos y tiendas de antigüedades de la ciudad, consultas en bibliotecas y búsqueda de documentos antiguos almacenados de manera física y digital. De la búsqueda entre el año 1925 y 1971, en los dos periódicos que presentaban perspectivas políticas extremas que lideraron en la época, siendo "El Derecho" el diario conservador y "El Radio" el diario liberal, se obtuvo información relevante que sirvió como base para la construcción posterior de la obra.

El segundo aspecto, consistió en la inclusión del diseño participativo en la investigación, el cual constó de entrevistas no estructuradas con historiadores, investigadores y expertos en estas épocas o temáticas para tener más claridad del contexto a estudiar, e igualmente con testigos para rescatar algunos relatos que se han perdido, ya que no se pueden encontrar en otras fuentes de información y que representan gran parte de las vivencias y a su vez están ligadas y componen la memoria histórica, recuperando sucesos del olvido.

A este aspecto también se sumó la elaboración de talleres, los cuales se realizaron con un grupo focal en el centro para el adulto mayor, La Edad Dorada, localizado en la ciudad de Pasto, con la participación de personas de la tercera edad, quienes interactuaron en diferentes actividades lúdicas, todas basadas en las temáticas principales de la investigación; actividades que se realizaron a manera de juego, con el fin de captar la mayor atención de los participantes. 


\begin{tabular}{|c|c|c|c|c|}
\hline \multirow{2}{*}{ 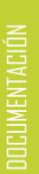 } & \multicolumn{2}{|r|}{ HERRAMIENTAS } & DESCRIPCIÍN & PRTDILTI \\
\hline & $\begin{array}{l}\text { - Indagación en fuentes } \\
\text { primarias y secundarias }\end{array}$ & $\begin{array}{l}\text { - NextStar Plus } \\
\text { - Voice Memos } \\
\text { - Microsoft Word }\end{array}$ & $\begin{array}{l}\text { Análisis del edificio y de la época de estudio } \\
\text { e identificación de los acontecimientos más } \\
\text { destacados por medio de textos y docu- } \\
\text { mentos, periódicos, planimetria, fotografías, } \\
\text { entrevistas y talleres participativos. }\end{array}$ & $\begin{array}{l}\text { - Identificación del telégrafo } \\
\text { como uso más relevante y de } \\
\text { las tres temáticas principales } \\
\text { que surgieron en dicha época. }\end{array}$ \\
\hline \multirow{2}{*}{ 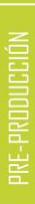 } & - Creación de la narrativa & - Microsoft Word & $\begin{array}{l}\text { Conceptualización de las tres temáticas } \\
\text { resultantes en un cuento dentro del marco } \\
\text { de la cotidianidad de las oficinas de correos } \\
\text { y telégrafos. }\end{array}$ & $\begin{array}{l}\text { - Narrativa } \\
\text { - Guión literario }\end{array}$ \\
\hline & $\begin{array}{l}\text { - Representación gráfica } \\
\text { de la narrativa }\end{array}$ & $\begin{array}{l}\text { - Dibujo análogo } \\
\text { - Adoble Illustrator } \\
\text { - Microsoft Excel }\end{array}$ & $\begin{array}{l}\text { Se plasma la narrativa en escenas que se } \\
\text { ajustan a características gráficas, } \\
\text { cinematográficas, arquitectónicas y } \\
\begin{array}{l}\text { conceptuales. }\end{array}\end{array}$ & $\begin{array}{l}\text { - Storyboard } \\
\text { - Guión técnico }\end{array}$ \\
\hline \multirow{3}{*}{ 를 } & $\begin{array}{l}\text { - Producción y edición } \\
\text { de contenido }\end{array}$ & $\begin{array}{l}\text {-Adobe Illustrator } \\
\text {-Adobe After effects } \\
\text {-Wondershare Filmora } 9 \\
\text { - Ableton live } 10 \\
\text { - Procesamiento analógico }\end{array}$ & $\begin{array}{l}\text { Elaboración de la pieza audiovisual a } \\
\text { proyectar, en calidad de animación y música, } \\
\text { teniendo como base el video de prueba } \\
\text { animatic. }\end{array}$ & $\begin{array}{l}\text { - Muestra de video animado: } \\
\text { - Animatic. } \\
\text { - Pieza musical vde la obra } \\
\text { - Pieza audiovisual: Animación } \\
\text { completa }\end{array}$ \\
\hline & $\begin{array}{l}\text { - Obtención del modelo } \\
\text { físico }\end{array}$ & $\begin{array}{l}\text { - Auto Cad } \\
\text { - Thunder Laser }\end{array}$ & $\begin{array}{l}\text { Se obtuvo mediante la elaboración de la } \\
\text { planimetría con las pertinentes medidas y } \\
\text { fotografías generales y de los detalles del } \\
\text { edificio. }\end{array}$ & $\begin{array}{l}\text {-Modelo físico del lugar: } \\
\text { maqueta del edificio Pasaje } \\
\text { Corazón de Jesús - escala 1:15 }\end{array}$ \\
\hline & - Instalación y mappeo & - MadMapper & $\begin{array}{l}\text { Acople de la obra audiovisual sobre la su- } \\
\text { perficie de la maqueta, ajuste de distancia y } \\
\text { angulación. }\end{array}$ & $\begin{array}{l}\text { - Proyección video mapping de } \\
\text { la pieza audio visual sobre la } \\
\text { fachada del edificio Pasaje } \\
\text { Corazón de Jesús }\end{array}$ \\
\hline
\end{tabular}

Tabla 1: Tabla de procesos.

En ellas se propició un espacio para conocer la vida personal de los integrantes y su cotidianidad, esto proporcionó una visión más clara del contexto, permitió retroalimentar la investigación y rescatar la memoria histórica a través de los relatos con fieles testigos de la época, obteniendo información que no se podría encontrar en ningún otro lugar más que en los recuerdos y vivencias de las personas que los presenciaron. De esta forma, además de hacer una inclusión a una comunidad subvalorada y excluida, se aprovecha una información de vital importancia como son los recuerdos que los testigos pueden brindar y que fueron la pieza clave para realizar la narrativa de la obra audiovisual.

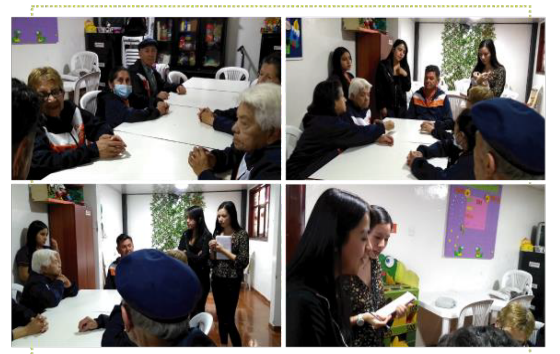

Figura 5: Talleres participativos.

\section{PERÍODO DE TIEMPO A ESTUDIAR: 1930 a 1963}

El proyecto se enfocó en el periodo de tiempo en el que el edificio funcionó como oficina de correos y telégrafos entre los años 1930 y 1963, de los cuales se hizo una previa contextualización social, cultural y política, ya que, en dicha época, surgieron acontecimientos importantes en cuanto a desarrollo, influencia política y cultural en la ciudad, que suponen un mayor aporte a la memoria del edificio.

\section{TEMÁTICAS PRINCIPALES}

Al finalizar el proceso de documentación se seleccionó la información más influyente y significativa dentro del periodo de estudio. En cuanto a la memoria histórica del lugar, se identificó un contexto claro de dicha época, de lo cual resultaron tres temas principales, los cuales son: el conflicto político, la lucha de la mujer y el progreso en cuanto a conectividad de la ciudad de Pasto.

Basándose en estos temas y teniendo como principal condicionante la selección de los acontecimientos enlazados a los procesos comunicativos del telégrafo, se hizo una reconstrucción de la memoria a través de la creación de una narrativa que conceptualizó dichas temáticas, resaltando las vivencias y experiencias de la época, proporcionándoles visibilidad y potenciando la valoración y la apropiación de las mismas.

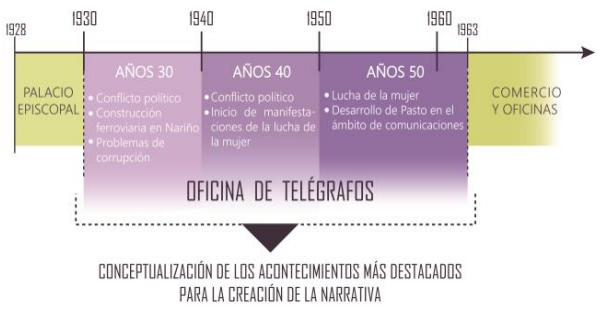

Figura 6: Línea de tiempo entre 1930 - 1963. 


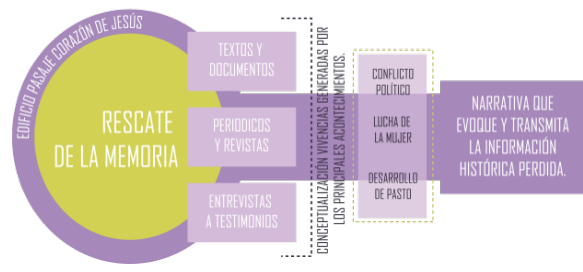

Figura 7: Proceso para el rescate de la memoria histórica.

\section{CONCEPTUALIZACIÓN}

\section{CONSTRUCCIÓN DE LA NARRATIVA}

Para la construcción de la narrativa, se partió de la conceptualización de las temáticas principales a modo de metáforas, las cuales dieron origen al concepto gráfico de la obra. Se inició con la elaboración de una premisa que aborda la idea general de la historia y que abarca tres décadas con diferentes temas, desde la cotidianidad de la oficina de telégrafos. A partir de este punto se realizó un cuento que relató cuatro microhistorias enfocadas en las temáticas de cada década, en las que se narran los sucesos más destacados en cada período de tiempo.

Teniendo en cuenta que la copiosa cantidad de información recopilada y su complejidad, se pensó en una forma de construir la narrativa de modo que fuera más sencilla y amigable en cuanto a su comprensión. Como estrategia se optó por la implementación de metáforas satíricas con el fin de que fueran más llamativas y abarcaran un público más extenso; como resultado surgieron representaciones tales como:

- Para los partidos políticos, con el fin de expresar la rivalidad entre sus seguidores, se optó por representar los personajes manejando los dos colores, azul y rojo, de los respectivos partidos que muestran la radicalidad con la que estos eran manejados; este conflicto también se simboliza por medio de dos tiburones que al combatir demuestran el único interés por adueñarse de la presa que representa a la población.

- En la lucha de la mujer se utilizan bolas de hierro atadas a sus tobillos que representan los obstáculos o desventajas que tenían ellas en comparación con los hombres en cuanto a liderazgo, desarrollo y especialmente libertad.

- El cambio de escala se usó en varias ocasiones para personificar el poder que puede tener un personaje sobre otro, o la relevancia que éste tiene según la actividad que esté desenvolviendo en cada escena; también fue utilizado para evidenciar algún tipo de injusticia o para enfatizar en las expresiones faciales con fines narrativos.

- De forma más conceptual, se utilizaron emblemas con las distintas ciudades de Colombia para representar la situación de aislamiento en la que se encontraba la ciudad de Pasto en la época de estudio y como se fue desenvolviendo a través de la llegada de los distintos canales de comunicación.

- Para los testimonios, se ilustraron las caras de los testigos de forma abstracta, representando a través de los rostros la imagen vívida de la memoria en el edificio y dándole realismo al relato.

- Se mostró el edificio simbolizando la ciudad de Pasto, con el objetivo de representar los problemas internos de los años 30's, develando el espacio interior del Pasaje y la relación de éste con los sucesos, plasmándolos de manera conceptual en cada piso, para crear una atmósfera espacial inmersiva que deje claro el contexto, por ejemplo, el ferrocarril como un personaje, representando su caso de corrupción.

\section{PREPRODUCCIÓN}

\section{GUIÓN LITERARIO}

Toda esta información se plasmó en un guión literario en el cual se ordenaron las historias en escenas y se estableció un tiempo de duración específico; también se

\section{PRRCESU DE CONCEPTUALIZACIÍN}

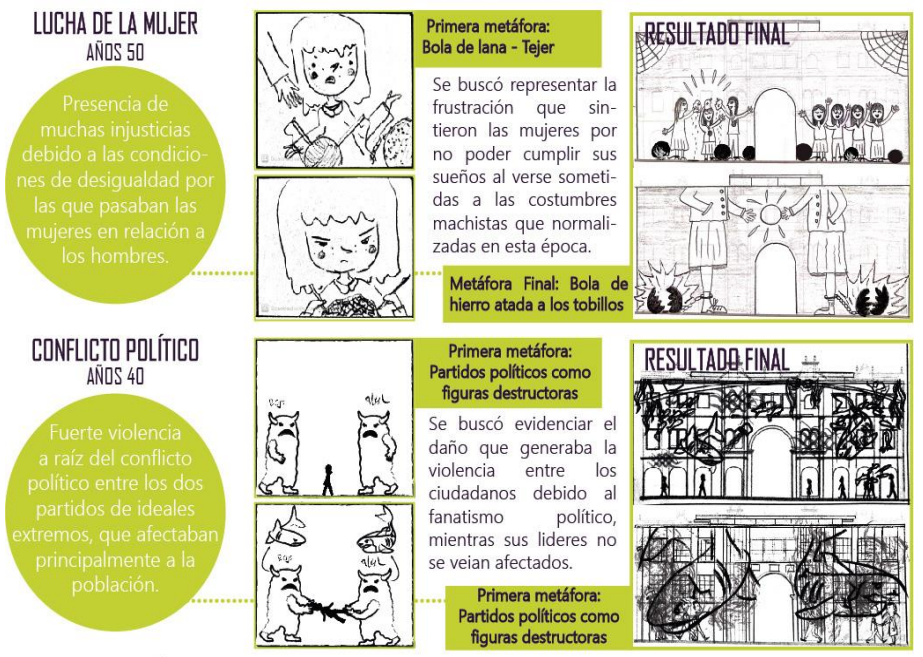

Figura 8: Proceso de conceptualización. 
determinó una estructura que ordena los elementos narrativos, haciendo una introducción general y posteriormente ubicando las microhistorias, dándoles un preámbulo y un final que contextualice sus condiciones. En este guión se detallaron algunos aspectos gráficos a considerar respecto a la fachada y cómo se utilizaron sus elementos dentro de la narrativa (figura 8).

\section{STORYBOARD}

Posteriormente se elaboró el storyboard, en el que se dibujaron cada una de las escenas en cuadros claves traduciendo la intensión visual de cada escena en una idea gráfica.

Se dibujaron las escenas sobre la fachada digitalizada resaltando la poética del edificio y se hizo homenaje a todos sus elementos y pequeños detalles, usándolos dentro de la narrativa y dándoles un papel dentro de la gráfica de las historias plasmando una atmósfera psico espacial haciéndola más inmersiva.

La metáfora se refuerza haciendo uso de herramientas cinematográficas como los planos, el movimiento y la angulación de la cámara, enmarcando las expresiones de los personajes, para comunicar la psiquis de los mismos y así transmitir las emociones que se construyen a través de la estética y los planos.

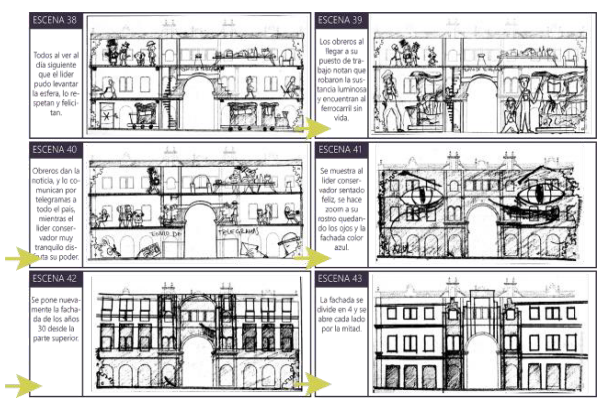

Figura 9: Representación gráfica de la narrativa.

\section{GUIÓN TÉCNICO}

Como antecedente del guión técnico se partió de la escritura del guión literario, en el cual se especificó la construcción y ubicación de cada escena y su duración en segundos. Posteriormente, se elaboró el guión técnico en el cual se anexó una descripción de la acción. En cuanto al sonido se incluyeron los fragmentos de las entrevistas, el tono de música específico, el sonido Foley y la emoción a transmitir. A la par se resaltaron las características arquitectónicas de cada escena, acompañado de un gráfico de la fachada teniendo en cuenta el análisis formal previamente hecho. Así mismo se consignó cada keyframe, que corresponde a un dibujo por escena del storyboard; adicionalmente se consignó la paleta de colores a utilizar, condicionada por la década y aplicada respectivamente a cada keyframe.

\section{PRODUCCIÓN}

\section{ANIMATIC}

Con las especificaciones anteriores se hizo una prueba traducida en un video animatic, conduciendo a una etapa de prueba y error, como estrategia para testear el ritmo de la obra hasta llegar a los tiempos deseados.

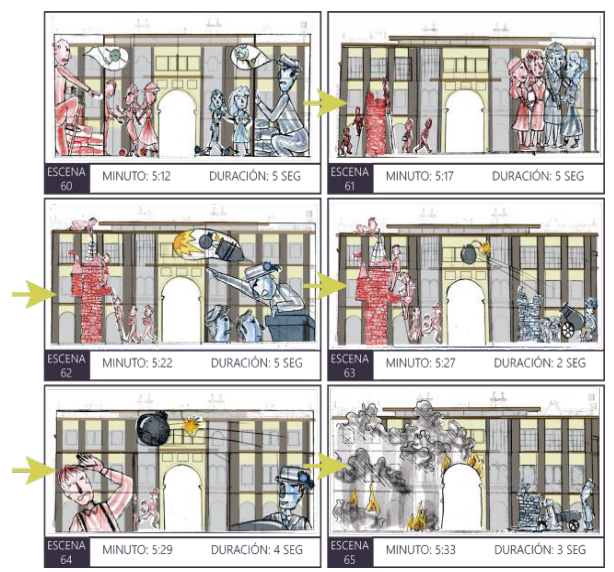

Figura 10: Video animatic - prueba de la obra.

Posteriormente se realizó un proceso de sincronización entre las ideas gráficas plasmadas en el storyboard y las condiciones geométricas de la fachada para iniciar con el proceso de animación en el que fue necesario realizar diferentes ajustes en la parte gráfica con la asesoría de los monitores Jheison Ramírez Pistala y Michael Narváez Muñoz, profesionales en diseño gráfico, con el fin de aprovechar la estética de la fachada a través de la exaltación de sus detalles, dando así más visibilidad a los mismos ante los ojos del público.

\section{ANIMACIÓN}

Posteriormente se siguió con el proceso de animación de las diferentes escenas según el orden establecido, teniendo una idea general del estilo gráfico basado en geometrías ortogonales de colores estridentes. Para el diseño de los personajes, se diseñaron los rostros con trazos haciendo uso de vectores y se representaron de forma abstracta según su propósito dentro de la narrativa, tomando elementos de la fachada. Finalmente se generó una ilustración minimalista en contornos dando sombras en alto contraste y agregando detalles en el movimiento a la hora de animar, teniendo como partida el boceto de los personajes y el entorno en el que estos se movieron. Posteriormente se vectorizaron los personajes teniendo en cuenta las articulaciones de los mismos y los objetos en movimiento. De este punto se partió para la creación de un esqueleto virtual en el cual se pueden anclar por partes los elementos en movimiento, dándoles directrices a través de comandos para realizar el desplazamiento deseado.

Simultáneamente se trabajó el proceso de creación de la 


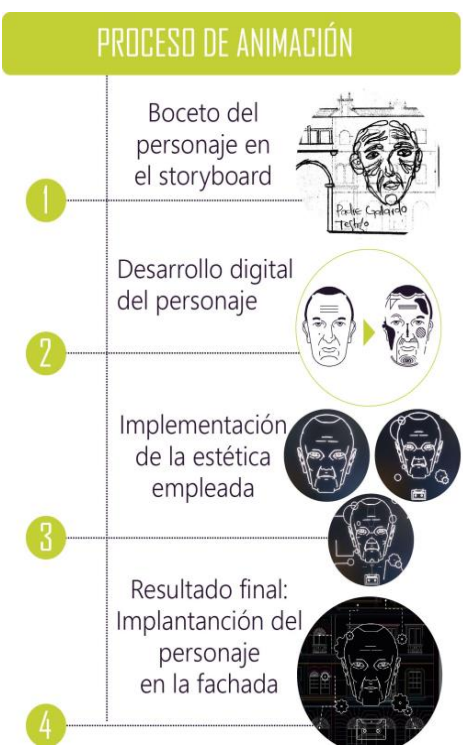

Figura 11: Diseño y animación de personajes.

obra musical que obedeció a las emociones e intensidades que debe transmitir cada escena, con el fin de obtener una obra armoniosa y coherente. Esta pieza musical se realizó teniendo en cuenta el sonido foley y la música, cuyos samples fueron grabados de manera analógica y posteriormente procesados en el software Ableton Live 10, en el que se produce la pieza musical.

Por otra parte, cabe resaltar que en el proceso de creación de la obra musical se realizaron varias iteraciones que partieron de un tono intrigante y nostálgico, acompañados por un tipo de música ambiental. Posteriormente, con el

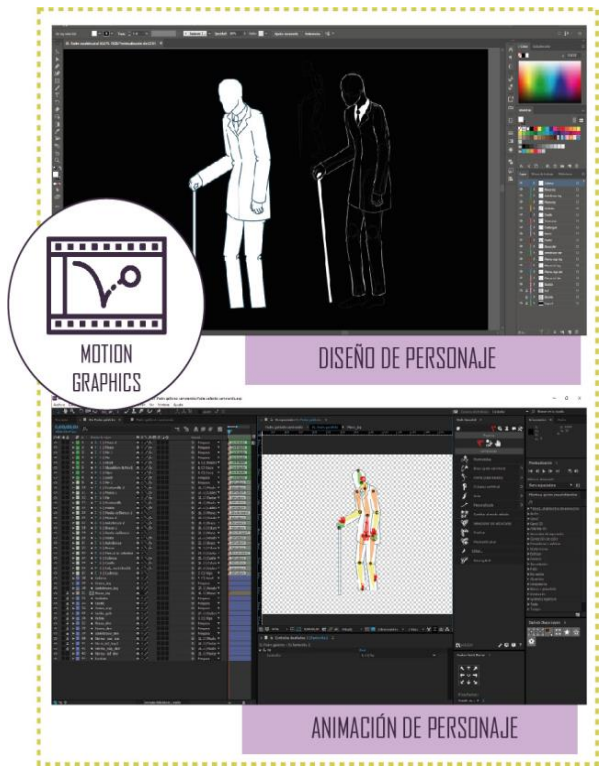

Figura 12: Diseño y animación de personajes a cargo de los animadores Jheison Ramírez y Michael Muñoz. avance gráfico de la propuesta, la obra transitó por matices de suspenso, rivalidad, frustración, entre otras, finalizando en la parte conclusiva con una atmósfera de tono emocionante y esperanzador. En este proceso de grabación de sonidos foley, musicalización y diseño sonoro contamos con la colaboración de Sebastián Montenegro y Sara Maya Montenegro, compositores y productores independientes. Los equipos utilizados para crear el sonido ambiental fueron sintetizadores análogos modulares y semimodulares y para la creación de las piezas sinfónicas se utilizaron instrumentos musicales análogos.

El proceso anteriormente mencionado se creó simultáneamente al de animación teniendo como principales factores el manejo de los tiempos, lo cual permitió la concordancia entre las emociones manejadas en cada escena y los movimientos presentes en la misma.

Cabe resaltar que para llevar a cabo la etapa de preproducción y producción del proyecto fue necesaria la participación y colaboración de diferentes disciplinas enfocadas al diseño para la creación de la obra audiovisual y la proyección de la misma como: diseño gráfico, artes visuales, música y arquitectura. La retroalimentación en los diferentes saberes ha permitido la exploración armoniosa y coherente en relación a la obra.

\section{ELABORACIÓN DE LA MAQUETA Y FASE EXPERIMENTAL DE MAPPEO}

Esta etapa corresponde al acoplamiento de la pieza audiovisual de Video Mapping con el medio físico, como es la maqueta de la fachada del edificio a escala 1:15, superficie sobre la que se proyecta la obra. Para esto se hizo acopio de la documentación planimétrica y fotográfica, para representar la fachada de acuerdo al diseño, estilo, dimensiones y materiales de la misma para así realizar una versión detallada y acorde para que la proyección logre realismo y permita apreciar cada detalle resaltando la poética de su fachada a lo que se le hizo oda y homenaje, evidenciando su arquitectura y estilo.

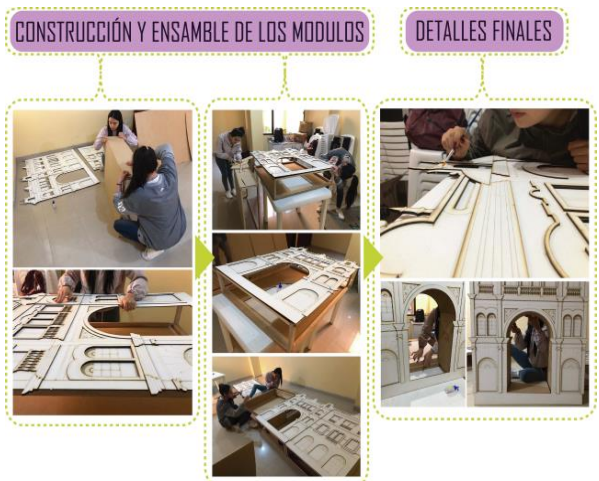

Figura 13: Elaboración del modelo esc 1:15.

Para llevar a cabo el proceso de mappeo, se inició con un testing, en el cual se realizaron pruebas que experimentan los diferentes aspectos que este conlleva, tales como la distancia, la angulación y la coherencia, entre otras, entendiendo el mecanismo y sus posibilidades. Para realizar estas pruebas fue necesario acoplar la obra audiovisual con la maqueta, cuyas dimensiones son de 
$2.30 \mathrm{~m}$ de ancho por $1.20 \mathrm{~m}$ de alto. Este proceso fue la primera aproximación que se tuvo entre la proyección y el medio físico a través del software MadMapper, en el cual se seccionó la fachada en cinco partes, para facilitar el acoplamiento en la fase de experimentación, en la que se ubicó cada fragmento del video en la parte correspondiente a la maqueta, resaltando detalles de la fachada que destacan su belleza arquitectónica y además permitiendo así tener una visión clara y perfeccionar la proyección.

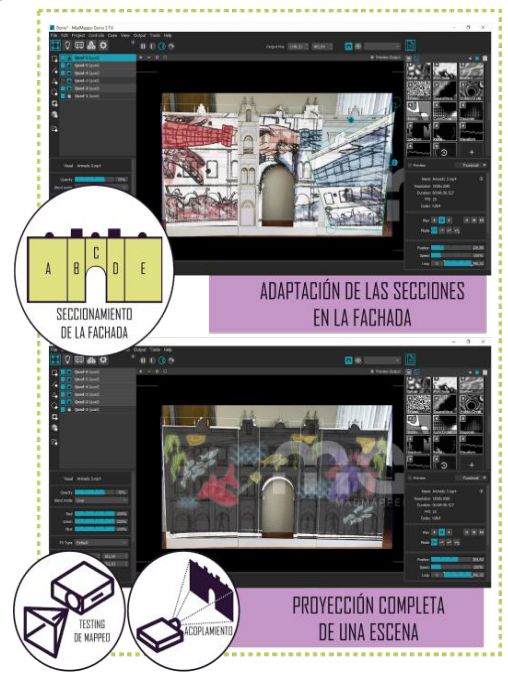

CONCLUSIONES Figura 14: Testing de mapeo.

- La presencia del edificio Pasaje Corazón de Jesús a pesar de sus años, historia, valor patrimonial y arquitectónico, se ha ido dejando a un lado y su situación en la plaza se ha normalizado, haciendo que pase desapercibido para los transeúntes y para los usuarios de la plaza, sin importar su belleza estética y el papel que tuvo en la historia de la ciudad.

- La ciudad de Pasto tiene una historia muy particular y compleja que no ha sido suficientemente registrada y custodiada. La ciudadanía ha sufrido procesos de desarraigo y la ausencia de cuidado en relación con la memoria histórica y cultural, ya que en su gran mayoría se encuentra extraviada o se ha ido perdiendo con el tiempo dejando vacíos enormes.

- Los talleres participativos son una pieza clave para la creación de la narrativa ya que brindan información que no se obtendría por ningún otro medio, por ser parte de los testimonios personales que nacen de la escucha de la palabra.

- Cada inmueble guarda en sí una memoria creada a partir de las vivencias que él mismo albergó, que a su vez cuentan una infinidad de historias, las cuales surgen de la relación que existe entre el lugar y quien lo habita lo cual aporta el foco sobre la dimensión humana de los espacios, a la par de su valor histórico y patrimonial.

- La unión y colaboración de diferentes disciplinas tales como: Arquitectura, Artes visuales, Diseño gráfico y Música, en simbiosis, tienen potencia para lograr resultados creativos en cuanto a contenido artístico y cultural, ya que, al integrarse y complementarse de forma armoniosa y coherente, pueden provocar y conmover desde su énfasis, sintetizados en la obra.

- Realizando el mapeo, se comprendió la importancia que conlleva el proceso de integración y coordinación de los diferentes paquetes de software de diseño y edición y de estos mismos con el medio físico sobre el cual se proyectará.

- El Video Mapping tiene potencia para comunicar un mensaje y por tanto logra ser transmisor de ideas y posibilitador de aperturas mentales, resultados que pueden ser explorados en cuanto a aspectos educativos, de promoción turística y de difusión patrimonial.

\section{DISCUSIÓN}

La memoria y el patrimonio arquitectónico de un edificio están ligados a su historia, pero normalmente se da más importancia a sus propiedades físicas y espaciales, excluyendo por completo las experiencias sensoriales. Este proyecto se enfoca y resalta la influencia que tiene la memoria como una dimensión fundamental de la arquitectura, reafirmando que: "El papel de la arquitectura, su tarea primordial, es generar interacciones entre el cuerpo, la mente y su entorno. Cualquier espacio proyectado tiene la capacidad de afectar nuestro cuerpo generando asociaciones a cada universo personal. Tiene el potencial de generar experiencias conmovedoras en el ser humano. Puede crear y evocar recuerdos. Tiene la capacidad de aparecer y hacer visible nuestro pasado, aquello que ya está ausente". (Camilo, 2016, p. 89). Entendiendo esto, se puede llegar a considerar el pensar la memoria como un factor casi independiente del patrimonio, que se define por variables, como el tiempo, el contexto social y por las experiencias personales y cómo estas se relacionan con el lugar, además de estar definidas por el mismo motivo, producto del uso, "La memoria requiere de un lugar donde acontecer porque la memoria es un diálogo complejo e indeterminado entre espacio y tiempo." (Sztulwark, 2005, p.3). Es así como la memoria vista desde este enfoque nos permite reconectar el pasado con el presente y devolverle relevancia, visibilidad y sentido.

\section{REFERENCIAS}

Botta, M. (2014). Arquitectura y memoria. Ra. Revista de Arquitectura, Universidad de Navarra. p. 91-98. Recuperado de: https://revistas.unav.edu/index.php/revista-dearquitectura/article/view/906

Enríquez, M., Ordóñez, A., Morales, H., Bolaños, A., \& Ortega, R. (2009). Espinoza y Santacruz: dos arquitectos dos épocas. Pasto: Impresiones Publimpacto.

Lafluf M., Barber G. (2017). Prácticas de la cultura digital y difusión del patrimonio. Video Mapping y remix.

Ordóñez, A.; Grupo de investigación ALARIFE. (2009). Espinosa y Santacruz. Pasto: Institución Universitaria CESMAG.

Sztulwark, P. (2005). Monumento, lugar y situación urbana. Ciudad memoria. p. 94- 97.

Camilo, I. (2016). Sobre la memoria y la arquitectura: construir la ausencia. Dearq. Revista de Arquitectura, (18), 80-87. 